\title{
Mycobacterial infection in an inner city children's hospital
}

\author{
H M Goodyear, J C Moore-Gillon, E H Price, V F Larcher, M O Savage, C B S Wood
}

Queen Elizabeth Hospital for Children, Hackney Road, London E2 8PS, Department of Paediatrics H M Goodyear V F Larcher M O Savage C B S Wood

Department of Microbiology E H Price

Department of Respiratory Medicine, St Bartholomew's Hospital, London J C Moore-Gillon

Correspondence to: Dr Goodyear.

Accepted 4 May 1993

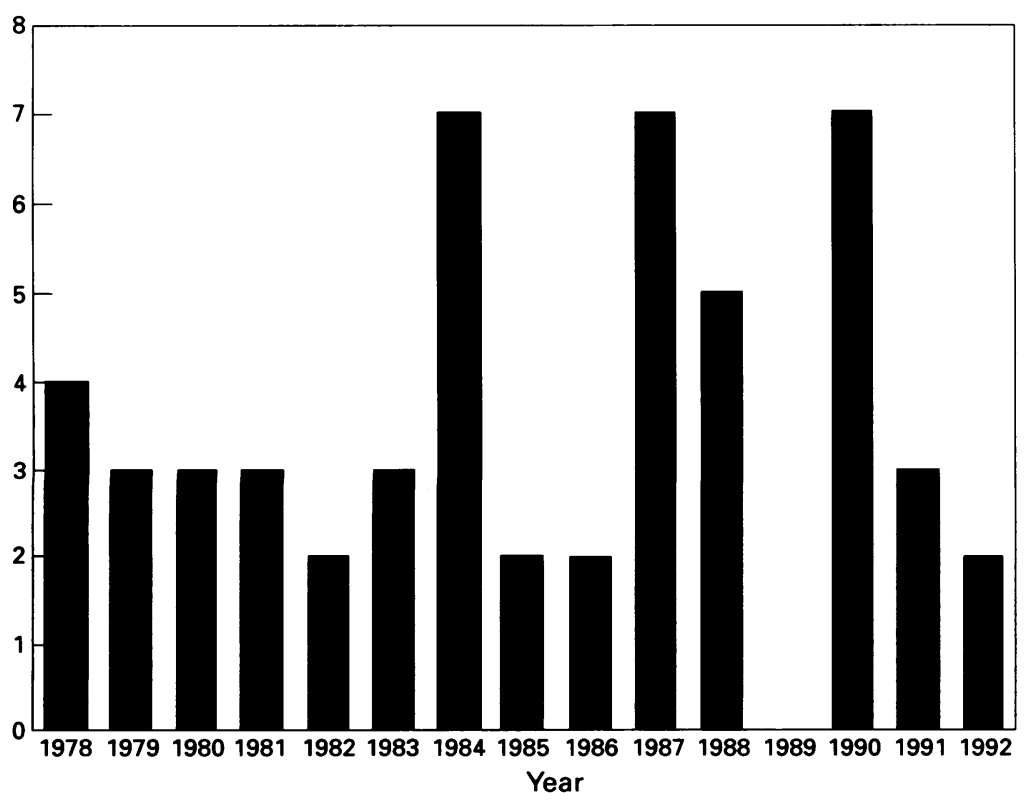

Figure 1 Cases of tuberculosis at QEH 1978-92.

\begin{abstract}
Childhood tuberculosis is perceived by many as a disease of the past. Experience in a children's hospital serving a deprived population suggested that tuberculosis and other mycobacterial infections were not declining in clinical practice.

Fifty three tuberculous and 11 atypical mycobacterial infections were identified between 1978 and 1992. There was no decline in tuberculosis and nine of the 11 atypical infections occurred in the last five years. Altogether $40 \%$ of cases of tuberculosis were in non-Asian children; $32 \%$ had arrived in the UK or visited family overseas in the previous year; and $38 \%$ had a history of tuberculosis contact, usually a close adult relative.

Nationally, the previous decline in tuberculosis in all ages has reversed. In the local health districts in London's east end, childhood tuberculosis has also stopped declining and seems to be increasing. It is regrettable that BCG vaccination has been abolished by some districts in the UK, against current recommendations. Childhood tuberculosis is still common in the practice described here, including among children who do not fall into conventionally recognised high risk groups. Inner city dwellers and junior doctors are both highly mobile populations, adding to the risk that paediatricians, particularly those in training, may encounter tuberculosis
\end{abstract} study.

with little or no previous experience of the condition.

(Arch Dis Child 1993; 69: 229-231)

Childhood tuberculosis has been considered to be a disappearing disease in Britain. ${ }^{1}$ The 1983 survey of tuberculosis notifications in England and Wales showed a $35 \%$ decline over 5 years, ${ }^{23}$ and on the assumption that this would continue the 1985 Department of Health and Social Security guidelines advised discontinuation of routine BCG vaccination of $10-14$ year olds. ${ }^{4}$

Our clinical experience led us to believe that the number of new cases of tuberculosis in our patients was not going down. Queen Elizabeth Hospital for Children (QEH) serves a socially deprived inner city catchment area in the east end of London (City and Hackney and Tower Hamlets health districts). This study examines all cases of mycobacterial infection diagnosed at the hospital from 1978 to 1992 .

\section{Patients and methods}

Cases were identified from the hospital coding system and microbiology department records. The diagnosis of mycobacterial infection was based on a positive culture, or clinical and radiological features suggestive of tuberculosis, in the presence of a positive tuberculin test. Children given isoniazid chemoprophylaxis for an isolated positive tuberculin test were not considered in this

\section{Results}

Sixty four cases of mycobacterial infection were identified from 1978-92: 53 tuberculous and 11 atypical mycobacterial infections. Tuberculosis has not declined at QEH over this 15 year period (fig 1 ). Fifteen cases of tuberculosis were seen in the first five years 1978-82, and 17 in the last five years 1988-92. Nine of the 11 atypical mycobacterial infections were seen in the last five years.

Our experience mirrors local trends; notifications of all cases of childhood tuberculosis in our local health districts 1983-92 are shown in fig 2, along with the QEH statistics. Before 1983, separate figures for childhood tuberculosis infections are not available for Hackney district. Notifications may indeed now be rising, showing a steady increase from 1989 (four cases) to 1992 (15 cases). This latest figure is the highest of the 10 years for which statistics are available. 


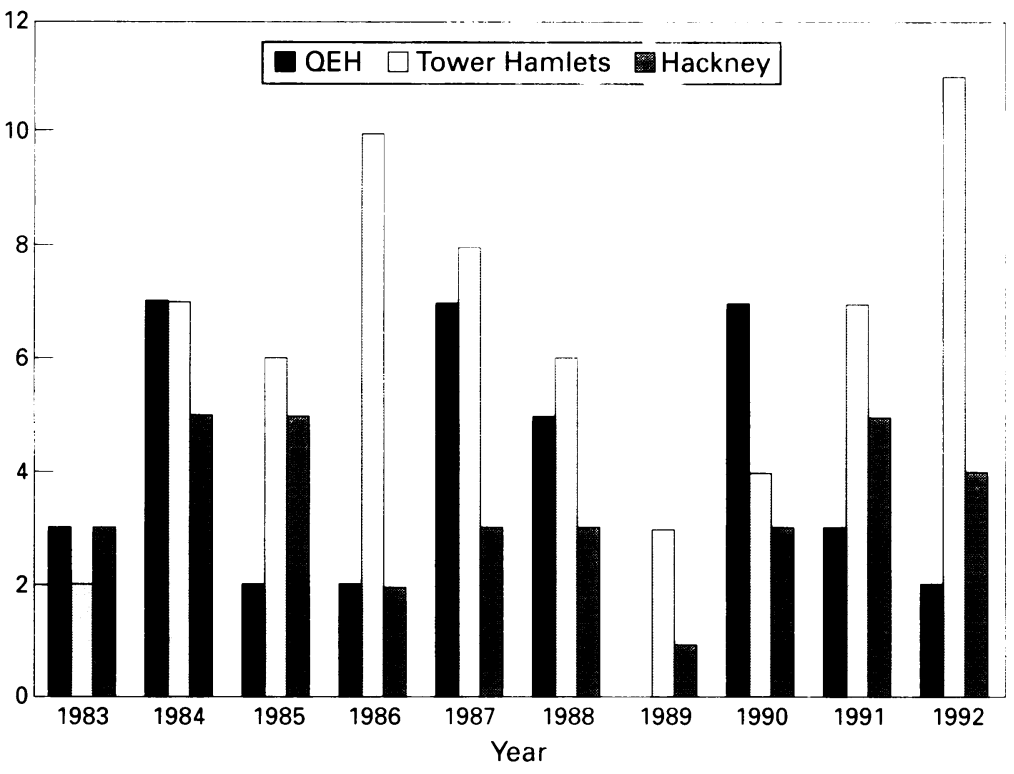

Figure 2 Cases of childhood tuberculosis at QEH compared with total notifications of childhood tuberculosis in the local health districts.

There were 28 boys and 25 girls in the 53 cases of tuberculosis seen at $\mathrm{QEH}$, aged 6 months to 13 years at diagnosis; $53 \%$ of cases were less than 5 years old. Forty eight children $(91 \%)$ presented as general practitioner outpatient referrals or parent initiated attendance at the accident and emergency department. The commonest complaints were cough, night fever, and weight loss. Thirty two children $(60 \%)$ were of Asian ethnic origin, 10 (19\%) Afro-Caribbean, eight $(15 \%)$ white, and three $(6 \%)$ non-Asian mixed race.

HISTORY OF CONTACT, BCG VACCINATION, AND FOREIGN TRAVEL

There was a history of tuberculosis contact, usually a close adult relative, in $20(38 \%)$ cases of tuberculosis, but only five $(9 \%)$ children were identified by contact tracing of other index cases. Ten children $(19 \%)$ had been vaccinated (nine Asian, one white), with only four $(8 \%)$ children receiving BCG vaccination in the neonatal period. Seventeen (32\%) children with tuberculosis had either visited Asia or arrived in the UK within one year of diagnosis.

\section{INFECTION SITES}

The principal sites of Mycobacterium tuberculosis infection were pulmonary and cervical lymph nodes accounting for $39(74 \%)$ and nine $(17 \%)$ cases respectively. The other affected sites were bone in four children $(8 \%)$, axillary lymph nodes in one $(2 \%)$, peritoneum in one $(2 \%)$, and pericardium in one child $(2 \%)$. Two children with tuberculosis had more than one site of infection.

The sites of atypical mycobacterial infection were cervical lymph nodes in six (55\%) cases, inguinal lymph nodes in one, and abscesses (one face, one left thigh, two buttock) in four children. All these children had a normal chest $x$ ray film.
BACTERIOLOGICAL FINDINGS

A positive culture for $M$ tuberculosis was obtained in $20(38 \%)$ cases of clinically diagnosed tuberculosis. Atypical mycobacterial infections were identified by culture as $M$ avium intracellulare $(\mathrm{n}=8), M$ chelonei $(\mathrm{n}=2)$, and $M$ scrofulaceum $(\mathrm{n}=1)$.

TREATMENT

All cases of $M$ tuberculosis were treated with conventional chemotherapy regimens. All atypical mycobacterial infections were treated by surgery. Chemotherapy with rifampicin and isoniazid for nine months was given in four $M$ avium intracellulare lymph node infections.

\section{Discussion}

The annual number of new cases of childhood tuberculosis at $\mathrm{QEH}$ has not diminished and our experience is representative of our local health districts. Although total notifications (child and adult) in England and Wales fell from 7406 in 1982 to 5085 in 1987, they have subsequently shown a slight increase (5357 mean notifications 1989-91).

It is important to be alert to the possibility of tuberculosis in all ethnic groups: $40 \%$ of our cases were in non-Asian children and residence in an urban priority area may be a better indicator of risk than ethnic group. ${ }^{5}$ Inner city populations can be highly mobile: population change in Hackney may be up to $30 \%$ per annum, a figure perhaps approached only by that of junior medical staff, who may thus encounter cases of tuberculosis having had little or no previous experience of the condition.

Contact tracing is vital to identify sources of infection, not just those to whom the child may have spread the disease. Twenty of the children treated by us had an infected close adult relative but only five of these had been identified by contact tracing. These apparently low numbers may be due to the fact that the local tuberculosis contact clinics are held at nearby hospitals, not at QEH. They are staffed by adult respiratory physicians, and at least some children are being treated without any input from paediatricians.

The increased risk of tuberculosis after visiting $\mathrm{Asia}^{6}$ and a higher infection rate in newly arrived immigrants ${ }^{7}$ is well documented. Recent visits to relatives in Asia by longstanding British residents must be remembered as a risk factor for tuberculosis by those investigating a sick child; $32 \%$ of our cases had immigrated or travelled abroad in the previous 12 months. Prompt identification and tuberculin testing of children newly arrived from abroad is essential. The statutory 'port of arrival' system is used in our local health districts in an attempt to ensure this, but it is known to be unreliable. ${ }^{8}$

The rising numbers of atypical mycobacterial infections in the last five years of the study is in accord with other reports of increased frequency, ${ }^{910}$ but our numbers are too small for statistical analysis. In purely localised 
lesions, resection is now the recommended treatment, ${ }^{91112}$ and some authors consider the use of chemotherapeutic agents is only necessary in disseminated, pulmonary, or bone atypical mycobacterial infections. ${ }^{10}$ All our cases were treated successfully, and there have been no recurrences (follow up 2-14 years).

The assumption that discontinuing routine BCG vaccination of schoolchildren would not give rise to an increase in tuberculosis (although it would slightly slow the annual rate of decline) was based on annual notification rates continuing to fall at a rate of around $9 \% .{ }^{13}$ In view of the lack of decline (and recent slight increase) in annual tuberculosis notifications, the wisdom of abolishing routine BCG must be questioned. Some health authorities are still doing so despite the most recent advice from the British Thoracic Society, which is to continue offering BCG vaccination to 10-14 year olds, and to neonates in areas where the incidence of tuberculosis is high. ${ }^{14}$ Since 1980 the two health authorities local to QEH have offered neonatal BCG vaccination to Asian and AfroCaribbean families, extended in 1984 in City and Hackney to neonates of all ethnic groups. Uptake has risen to about $60 \%$, but early discharge policies after childbirth create practical difficulties. Altogether $81 \%$ of our cases had not been vaccinated, and $40 \%$ were in non-Asian children.

Childhood tuberculosis remains an important problem. In our inner city hospital, cases are being seen with the same frequency now as they were over a decade ago. Paediatricians, particularly those whose early training has been in areas where the incidence of tuberculosis is low, must maintain a high index of suspicion for the disease.

1 Silverman M. Childhood tuberculosis in Britain. BMF 1988; 297: 1147-8.

2 Medical Research Council Tuberculosis and Chest Diseases Unit. Tuberculosis in children in a national survey of notifications in England and Wales, 1978-79. Arch Dis Child 1982; 57: 734-71.

3 Medical Research Council Tuberculosis and Chest Diseases Unit. Tuberculosis in children: a national survey of Unit. Tuberculosis in children: a national survey of notifications in England
Child 1988; 63: 266-76.

4 Department of Health and Social Security. The school $B C G$ vaccination programme. London: DHSS, 1985 (DA(85)27).

5 Cundall DB, Pearson SB. Inner city tuberculosis and immunisation policy. Arch Dis Child 1988; 63: 964-6.

6 McCarthy OR. Asian immigrant TB - the effect of visiting Asia. British fournal of Diseases of the Chest 1984; 78: 348-53.

7 British Thoracic and Tuberculosis Association. Tuberculosis among immigrants relating to length of Tuberculosis among immigrants relating to length of

8 Ormerod LP. Tuberculosis screening and prevention in new immigrants 1983-88. Respir Med 1990; 84: 269-7

9 Taha AM, Davidson PT, Bailey WC. Surgical treatment of atypical mycobacterial lymphadenitis in children. Pediatr Infect Dis 1985; 4: 664-7.

10 Margileth AM. Management of non tuberculous (atypical) mycobacterial infections in children and adolescents. Pediatr Infect Dis 1985; 4: 119-22.

11 White MP, Bangash H, Goel KM, Jenkins PA. Nontuberculous mycobacterial lymphadenitis. Arch Dis Child tuberculous mycoba

12 Schuit KE, Powell DA. Mycobacterial lymphadenitis in childhood. Am $\mathcal{F}$ Dis Child 1978; 132: 675-8.

13 Springett VH, Sutherland I. BCG vaccination of schoolchildren in England and Wales. Thorax 1990; 45: 83-8.

14 Joint Tuberculosis Committee of the British Thoracic Society. Control and prevention of tuberculosis in Britain: an updated code of practice. BMF 1990; 300: 995-9. 